Chapter 8

\title{
Testing Physical and Mathematical Criteria in a New Meandering Autocorrelation Function
}

\author{
Charles R.P. Szinvelski, Lidiane Buligon, \\ Michel Baptistella Stefanello, Silvana Maldaner, \\ Debora R. Roberti and Gervásio Annes Degrazia
}

Additional information is available at the end of the chapter

http://dx.doi.org/10.5772/67920

\begin{abstract}
An alternative formulation for the low wind speed-meandering autocorrelation function is presented. Employing distinct theoretical criteria, this mathematical formulation, from a physical point of view, is validated. This expression for the meandering autocorrelation function reproduces well-observed wind-meandering data measured in a micrometeorological site located in a pampa ecosystem area (South Brazil). The comparison shows that the alternative relation for the meandering autocorrelation function is suitable to provide meandering characteristic parameters. Employing MacLaurin's series expansion of a lateral dispersion parameter that represents cases in which turbulence and oscillatory movements associated to the meandering events coexist, a new formulation for the turbulence/meandering dissipation rate has been presented.
\end{abstract}

Keywords: wind meandering, meandering autocorrelation function, physical and mathematical criteria, turbulent energy spectrum, meandering period

\section{Introduction}

The autocorrelation function is a physical quantity important both for diffusion investigation and to provide basic relations concerning the turbulence processes in the planetary boundary layer (PBL). The employment of autocorrelation functions derived from observed data for distinct movement patterns in the PBL allows to evaluate relevant quantities used to understand complex phenomena in geophysical flow. Therefore, the utilization of the autocorrelation function in the Taylor statistical diffusion theory provides directly the dispersion parameters 
and also formulations for the turbulence dissipation rate, which are utilized, respectively, in Eulerian (Gaussian diffusion plume) and Lagrangian dispersion models.

Simulating turbulent diffusion and species scalar and vector transport in low wind speed situations is a challenging problem. For conditions, in which the mean wind speed tends to be negligible [1], it is very difficult to identify a defined mean wind direction. In this case, large-scale motions, such as submeso motions, control, in a dominate manner, the atmospheric contaminant dispersion [2]. These large horizontal wind oscillations are called meandering and are responsible for the fact that measured autocorrelation functions of the horizontal wind velocity components show a looping behavior, characterized by the presence of negative lobes [3]. This looping pattern with the presence of negative lobes in autocorrelation functions, which characterize the meandering observed phenomenon, can be well reproduced by particular mathematical formulations. With this good fit, it is possible to estimate the principal physical variables that specify the meandering flow [4,5].

Recently, Moor et al. [6] proposed to utilize a new mathematical formulation to fit the observed low wind speed-meandering autocorrelation functions. This functional form for the horizontal wind velocity components $u$ and $v$ may be written as follows:

$$
R(\tau)=\frac{\cos \left(\frac{m \tau}{\left(m^{2}+1\right) T_{L}}\right)}{\left(1+\frac{\tau}{\left(m^{2}+1\right) T_{L}}\right)^{2}}
$$

where $\tau$ is the time lag, $T_{L}$ is the decorrelation time scale, and $m$ is the loop parameter. The mathematical formulation, as provided by Eq. (1), is composed of the product of the binomial function (describing the autocorrelation function for the turbulent degrees of freedom [7]) by the cosine (describing the influence of the submeso motions in the autocorrelation function [8]). Therefore, this formulation presents a heuristical flexibility that allows describing the observed characteristics of the atmospheric multiple-scale interaction. Eq. (1) can also be written in a distinct way, namely

$$
R(\tau)=\frac{\cos (\mathrm{q} \tau)}{(1+\mathrm{p} \tau)^{2}}
$$

with

$$
p=\frac{1}{\left(m^{2}+1\right) T_{L}}
$$

and

$$
q=\frac{m}{\left(m^{2}+1\right) T_{L}}
$$

$q$ and $p$ are hybrid relations described in terms of the turbulent $\left(T_{L}\right)$ and meandering parameters $(m)$ and are originated from the Frenkiel autocorrelation function [9]. Furthermore, a meandering characteristic time scale (meandering period) can be represented as 


$$
T^{*}=\frac{2 \pi}{q}
$$

and will be calculated by

$$
T^{*}=\frac{2 \pi\left(m^{2}+1\right) T_{L}}{m}
$$

The purpose of this study is to verify that the expression given by Eq. (1) fills the mathematical and physical requirements that must be fulfilled by an autocorrelation function. An additional aim is to employ the best-fitting curves, obtained from Eq. (1), to calculate the loop parameter and the meandering characteristic time scale. The present investigation also provides a new relation for the turbulence/meandering dissipation rate.

\section{Discussion of the physical and mathematical criteria to the autocorrelation function}

In this part of the chapter, we use four established criteria [10] to validate the autocorrelation function, as given by the mathematical formulation described in Eq. (1). Therefore, these criteria are applied to stationary and homogeneous turbulence, which are mathematical descriptions of respective physical requirements for the validation of the autocorrelation function applied to the studies of turbulence. Eq. (1) is described in terms of a parameter $m$, which indicates the intensity of the meandering phenomena. This parameter is responsible to introduce variations in the usual results that are those normally expected in the classical forms that represent the autocorrelation functions. In sequence, the four criteria are discussed in detail as follows:

(I) $R(\tau)$ is an even function, and it has the following properties:

a. $|R(\tau)| \leq=R(0)$, it is limited in the origin and its maximum value 1 occurs in $\tau=0$. This fact agrees with the theoretical consideration presented in $[11,12]$. The authors suggest that $R$ must osculate the theoretical parabolic form of $R$ in the origin $(\tau \approx 0)$.

b. The next property takes into account the qualitative behavior to large values of autocorrelation time. In this case, $R(\tau) \rightarrow 0$ when $|\tau| \rightarrow \infty$. In Eq. (1), this consideration results of confront theorem applied in $|R(|\tau|)| \leq(1+p|\tau|)^{-2}$;

c. To define the integral time scale, it is necessary to calculate the integral $\int_{0}^{\infty} R(\tau) d \tau$, namely the integral must be convergent. Thus,

$$
\int_{0}^{\infty} R(\tau) d \tau \leq\left|\int_{0}^{\infty} R(\tau) d \tau\right| \leq \int_{0}^{\infty}|R(\tau)| d \tau=\frac{1}{p}=\left(m^{2}+1\right) T_{L}
$$

is a finite value. 
(II) $R(\tau) \in C^{0}(\mathfrak{R})$, wherever the smoothness is not valid in $\tau$ because the lateral derivatives in origin are not defined. This fact yields the angular functional form of the autocorrelation function to $\tau=0$, and its physical implication is that this autocorrelation function is not suitable to investigate the viscous microstructure of turbulence [13]. Therefore, to practical applications, where the hypothesis of fully developed turbulence is admitted $(\operatorname{Re} \gg 0)$, the validity of this criteria is not relevant by the fact that the energy-containing and the characteristics scales of eddies in this region are not relevant in the scalar diffusion process.

(III) As already mentioned, the integral time scale $T_{L}$ is well defined and its value is obtained as $\int_{0}^{\infty} R(\tau) d \tau$. Here, it is necessary to make some considerations:

- the integral resolution is ${ }^{1}$

$$
\begin{gathered}
\int_{0}^{\infty} \frac{\cos (q \tau)}{(1+p \tau)^{2}} d \tau=\left(1+m^{2}\right) T_{L}\left[1-m\left(\sin (m) \operatorname{Ci}(m)-\cos (m)\left(\operatorname{Si}(m)-\frac{\pi}{2}\right)\right)\right] \\
=\left(1-\beta_{\mathrm{m}}\right)\left(1+m^{2}\right) T_{L}
\end{gathered}
$$

- to $m=0$, the result obtained is $T_{L}$, in agreement with [7] and attends the given interpretation of [10] to these criteria, and, following [14], this result is expected because the argument of the autocorrelation function has dimensions of length inverse and after integration recovers dimensions of integral scale;

- but to $m \neq 0$. There is the factor $\left(1-\beta_{\mathrm{m}}\right)$, which goes to zero when $m$ goes to infinity. This fact tells us that it does not have significant correlation to large values of $m$;

Therefore, using the above considerations, this result suggests the following interpretation: the case $m=0$ will establish the autocorrelation time $T_{L}$ to a fully developed turbulence hypothesis. On the other hand, when $m \neq 0$, the time scale will be proportional to $T_{L}$ by the following correction factor $\left(1-\beta_{\mathrm{m}}\right)$ yielding

$$
T_{L m}=\left(1-\beta_{\mathrm{m}}\right)\left(1+m^{2}\right) T_{L}
$$

(IV) To validate these criteria, basically, we must verify the following relation [15]:

$$
E(\omega)=\frac{2\left\langle u^{2}\right\rangle}{\pi} \int_{0}^{\infty} R(\tau) \cos (\omega \tau) d \tau \approx \omega^{-2}
$$

\footnotetext{
${ }^{1} \mathrm{Ci}(m)$ is the cosine integral function and $\mathrm{Si}(m)$ is the sine integral function.
} 
In this case, applying the Fourier transform in Eq. (1) results

$$
\begin{aligned}
\frac{E(\omega)}{\left\langle u^{2}\right\rangle T_{L}}= & \frac{\left(m^{2}+1\right)}{\pi}\left\{2-A\left[\cos (A)\left(\frac{\pi}{2}-\operatorname{Si}(A)\right)+\sin (A) \operatorname{Ci}(A)\right]\right. \\
& \left.-B\left[\cos (B)\left(\frac{\pi}{2}-\operatorname{Si}(B)\right)+\sin (B) C i(B)\right]\right\}
\end{aligned}
$$

with $A=\left(m^{2}+1\right)\left(\omega T_{L}\right)+m$ and $B=\left(m^{2}+1\right)\left(\omega T_{L}\right)-m$.

Eq. (11) does not express the form $\omega^{-2}$, explicitly, but this behavior is founded in its graphic representation (see Figure 1).

The present analysis, based on mathematical and physical criteria extensively discussed in the literature, showed that the meandering autocorrelation function, as given by Eq. (1), can be employed to investigate the physical patterns associated to the meandering multiplescale flow.

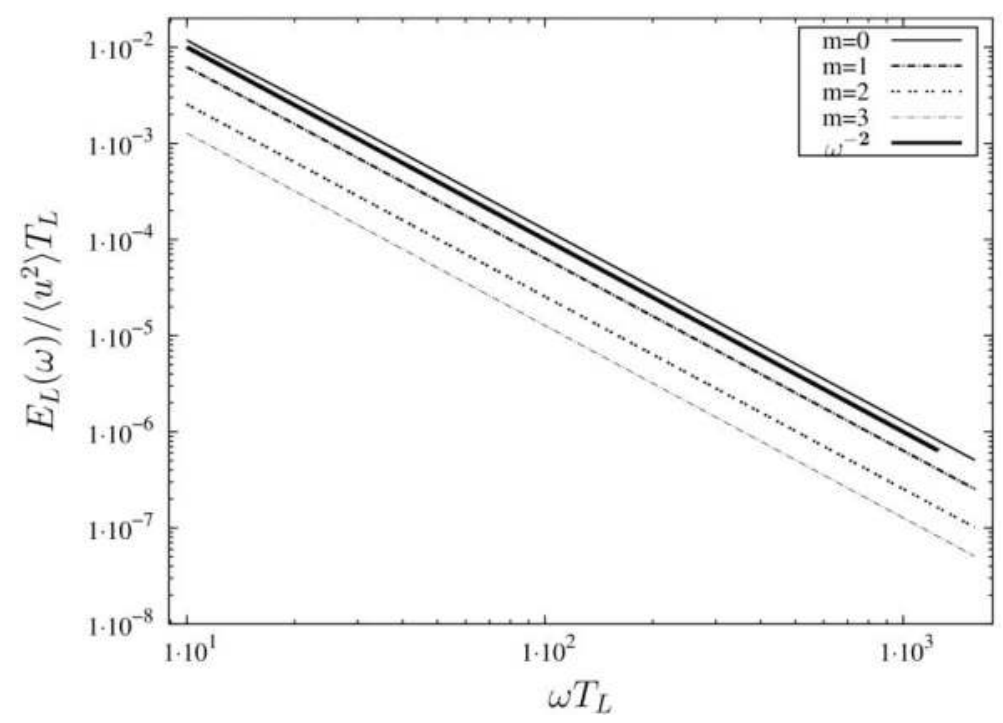

Figure 1. Turbulent energy spectrum from the autocorrelation functions (Eq. (1)) for distinct values of the $m$ parameter.

\section{Reproducing observed meandering autocorrelation functions}

In this part of the study, we use meandering data observed in a low wind speed PBL to calculate the experimental autocorrelation functions. These experimental meandering autocorrelation functions were obtained from the following relation: 


$$
R(\tau)=\frac{\langle u(t) u(t+\tau)\rangle}{\left\langle u^{2}\right\rangle}
$$

where $\left\langle u^{2}\right\rangle=\sigma_{u}^{2}$ is the variance of the velocity. These observed functions are fitted by Eq. (1). Therefore, Eq. (1) is evaluated as a mathematical representation to reproduce the observed negative lobes in the meandering autocorrelation functions and utilized to calculate the loop parameter and the meandering period.

The low wind speed data were collected at the Federal University of Santa Maria (Rio Grande do Sul, Brazil) meteorological site. The wind velocity components were sampled at a frequency of $10 \mathrm{~Hz}$ by a sonic anemometer installed at a height of $3 \mathrm{~m}$ located in a pampa ecosystem area.

Figures 2 and 3 establish the comparison among the autocorrelation function obtained from the low wind speed data set (continuous line) and the correspondent best fit (dashed line)

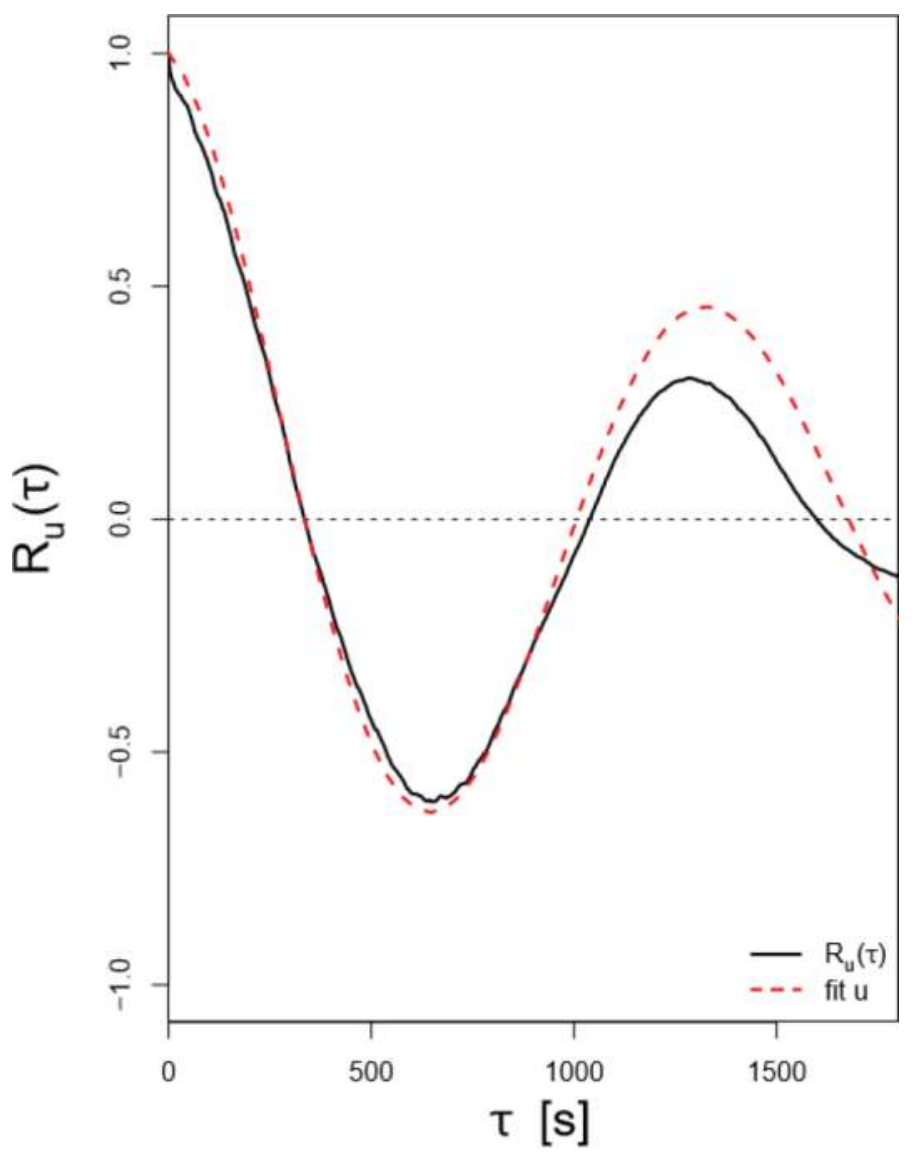

Figure 2. Autocorrelation function for the horizontal wind-velocity component $u$. 


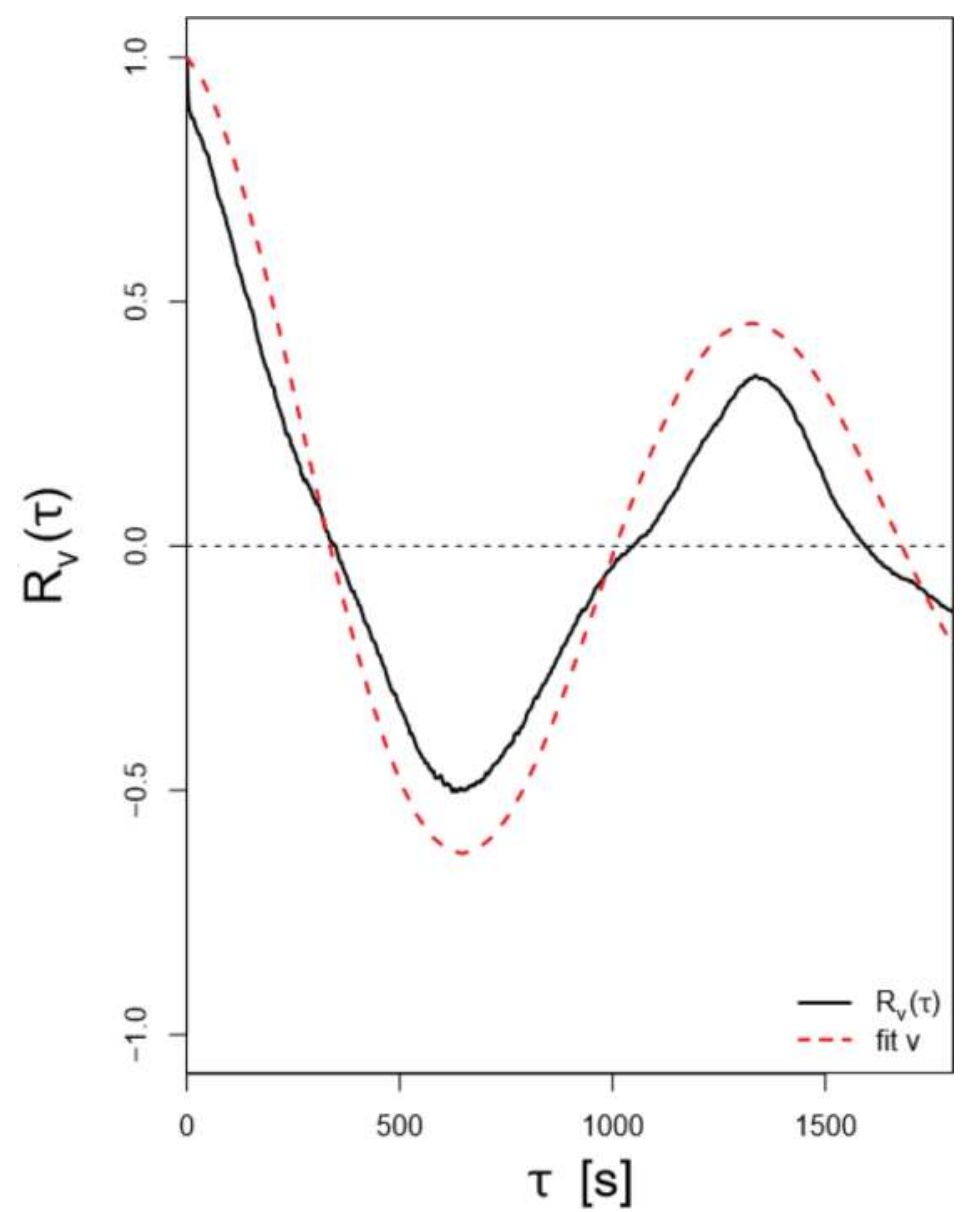

Figure 3. Autocorrelation function for the horizontal wind-velocity component $v$.

provided from Eq. (1). These figures were calculated from 1-h time series presenting the mean horizontal speed $\left(\bar{u}=0.96 \mathrm{~ms}^{-1}\right)$. They show that the autocorrelation function, provided by the mathematical formulation (Eq. (1)), represents fairly well the observed form of the meandering autocorrelation functions including their negative lobes. This oscillatory behavior, generating negative lobes in the autocorrelation functions, calls attention for the presence of multiple scales in the horizontal wind-meandering flow. The action of the submeso [8] and turbulent scale motions provoke, for short-time intervals, large variations in the horizontal wind direction.

Employing Eqs. (3)-(5) and a best-fit curve condition, it is possible to calculate, respectively, the magnitudes for the looping parameter and meandering period (a complete discussion about this development can be seen in [1]). These values are presented in Table 1. 
The magnitudes for $m_{u}$ and $m_{v}$ in Table 1 agree very well with the results obtained by Moor et al. [6] that employed wind-meandering data which were collected in a nocturnal stable PBL in a Brazilian Amazon Large Scale Biosphere-Atmosphere Project. The meandering periods $T_{u}^{*}$ and $T_{v}^{*}$ in Table 1 present similar magnitudes with the mean values found by [4]. The characteristic values of the meandering phenomenon are exhibited in Table 1, which can be used in Eqs. (6) and (9) to obtain the new turbulent scales, yielding $T_{L, m_{u}} \approx 70 \mathrm{~s}$ and $T_{L, m_{v}} \approx 62 \mathrm{~s}$. These magnitudes of $T_{L, m}$ are in agreement with the results estimated by Moor et al. [6].

\begin{tabular}{llll}
\hline$T_{u}^{*}(s)$ & $T_{v}^{*}(s)$ & $m_{u}$ & $m_{v}$ \\
\hline 1337 & 1337 & 5.2 & 4.2 \\
\hline
\end{tabular}

Table 1. Meandering period and loop parameter values for the $u$ and $v$ components.

\section{Turbulence/meandering dissipation rate}

The lateral dissipation parameter $\sigma_{y}$ is a statistical quantity fundamental for the dispersion modeling and for the derivation of turbulence/meandering dissipation rate functional form $[16,17]$. From the Taylor statistical diffusion theory, this relevant parameter may be described as

$$
\sigma_{y}^{2}(t)=2 \sigma_{v}^{2} \int_{0}^{t}(t-\tau) R_{v}(\tau) d \tau
$$

where $\sigma_{v}$ is the standard deviation of the turbulent lateral velocity.

Taylor [18] considered an exponential form for the autocorrelation function and first derived an expression for $\sigma_{y}^{2}$

$$
\sigma_{y}^{2}(t)=2 \sigma_{v}^{2} T_{L v}^{2}\left(\frac{t}{T_{L v}}-1+e^{\frac{-t}{L v}}\right)
$$

where $t$ is the travel time of the fluid particle, $T_{L v}$ is the lateral Lagrangian integral time scale (defined in Criteria III).

Tennekes [16] used Eq. (14) and inertial subrange similarity arguments [15, 12] to derive the following fundamental expression for the turbulence dissipation rate $\varepsilon$ :

$$
\varepsilon=\frac{2}{C_{0}} \frac{\sigma_{v}^{2}}{T_{L v}}
$$

where $C_{0}$ is the Kolmogorov constant.

Following the above development and employing the autocorrelation function as given by Eq. (1) into Eq. (13) yields 


$$
\begin{aligned}
\frac{\sigma_{y}^{2}(t)}{2 \sigma_{v}^{2}}=-\frac{\cos (q t)}{p^{2}} & +\frac{1+p t}{p^{2}}-\frac{q(1+p t)}{p^{3}}\left[\sum_{n=0}^{\infty}\left(\frac{(p t)^{2 n+2}}{2 n+2}-\frac{(p t)^{2 n+3}}{2 n+3}\right) \sum_{k=0}^{n} \frac{(-1)^{k} m^{2 k+1}}{(2 k+1) !}\right] \\
& -\frac{1}{p^{2}}\left[\sum_{n=0}^{\infty}\left(\frac{(p t)^{2 n+1}}{2 n+1}-\frac{(p t)^{2 n+2}}{2 n+2}\right) \sum_{k=0}^{n} \frac{(-1)^{k} m^{2 k}}{(2 k) !}\right]
\end{aligned}
$$

For $t \ll T_{L v}$, MacLaurin's series expansions of the meandering and turbulent dissipation parameter (Eq. (16)) can be approximated by

$$
\sigma_{y}^{2}(t)=\sigma_{v}^{2} t^{2}-\frac{2 p \sigma_{v}^{2}}{3} t^{3}
$$

The confrontation of Eq. (17) with the Taylor statistical diffusion theory (Eq. (13)), for dispersion periods that are very smaller than the Lagrangian integral time scale, demonstrates that the negative expression in Eq. (17) represents a term that reduces the hybrid dispersion parameter. This negative contribution results in the suppression of a number of degrees of freedom of the turbulent field associated to the high-frequency harmonics [17]. As a consequence, it is reasonable to relate the term $\frac{2 p \sigma_{v}^{2}}{3} t^{3}$ to the Kolmogorov spectrum high-frequency eddies. This comparison was accomplished by [16] employing the Lagrangian structure function, the Lagrangian autocorrelation function, and the inertial subrange Lagrangian turbulent spectrum. Tenneke's derivation provides the following relationship for the lateral dispersion parameter in terms of inertial subrange quantities:

$$
\sigma_{y}^{2}(t)=\sigma_{v}^{2} t^{2}-\frac{C_{0} \varepsilon}{6} t^{3}
$$

The comparison of Eq. (17) with Eq. (18) leads to the following fundamental relation:

$$
\varepsilon=\frac{4}{\left(m^{2}+1\right)} \frac{\sigma_{v}^{2}}{C_{0} T_{L v}}
$$

The new expression, as given by Eq. (19), maintains the basic argument that turbulent motion is dissipated at a rate proportional to the kinetic energy and inversely proportional to the memory effect associated to the energy-containing eddies. Therefore, Eq. (19) is described in terms of the looping parameter $m$. Such a parameter, associated to the meandering phenomenon, becomes the new dissipation rate distinct of those applied to the purely turbulent cases. The looping parameter $m$ determines the magnitudes of the dissipation rate. Hence, the dissipation rate increases when the turbulence is dominant. On the other hand, the dissipation rate decreases when submeso motions, associated to the meandering phenomenon, control the geophysical flow. 


\section{Conclusion}

In this study, a mathematical relation to represent observed meandering autocorrelation functions, derived from the heuristic arguments, is tested and validated using well-known mathematical and physical criteria. Therefore, it is employed to describe experimental windmeandering data. From this approach and utilizing best-fitting curves, it was possible to obtain characteristic values for the loop parameter and the meandering period. These quantities, which characterize the wind-meandering phenomenon, are shown in Table 1.

An important aspect in the present development has been the derivation of Eq. (11) which represents a theoretical formulation to model observed wind-meandering spectral data. Thus, Eq. (8) is able to describe the effect of the submeso and turbulent scales and hence allow to identify the low frequencies associated to the meandering spectral peaks.

Another relevant result is provided by Eq. (19). Therefore, this expression is able to evaluate dissipation rates generated from complex flows. Complex flows, such as meandering motions, are those in which there are interactions between distinct circulations characterized by movements presenting different spatial and time scales. As a consequence, Eq. (19) allows to describe this atmospheric phenomenon type.

\section{Acknowledgements}

The authors would like to thank the CNPq (Conselho Nacional de Desenvolvimento Científico e Tecnológico) and CAPES ( Coordenação de Aperfeiçoamento de Pessoal de Nível Superior) for partial financial support of this work.

\section{Appendix A}

In this appendix, the general criteria to validate mathematical formulation for the autocorrelation function are presented. These criteria are a summary of physical and mathematical requirements applied in the statistical treatment of the turbulence founded in the classical literature $[10,13]$.

In particular, Manomaiphiboon and Russell [10] presented four criteria for the validation of the ACF to homogeneous and stationary turbulence, as follows:

(I) $R(\tau)$ is limited at origin (neighborhood of origin) $|R(\tau)| \leq 1=R(0)$. Besides, $\lim _{|\tau| \rightarrow \infty} R(\tau)$ $=0$ and $\int_{0}^{\infty}|R(\tau)| d \tau<\infty$.

In fact, the generic form of the autocorrelation function is given by the Eq. (12)

following $R(0)=1$, which indicates that the maximum correlation is $\tau=0$ and, consequently to any other correlation time, is given by $|R(\tau)| \leq 1$. 
On the other hand, the condition that $\int_{0}^{\infty}|R(\tau)| d \tau$ must be finite will permit to obtain the Lagrangian integral scale time, which will be defined in Criteria III.

(II) $R(\tau)$ is smooth in $\tau$. At origin $\left.\frac{d R(\tau)}{d \tau}\right|_{\tau=0}=0$ and $\left.\frac{d^{2} R(\tau)}{d \tau^{2}}\right|_{\tau=0}<0$.

The mathematical properties described by Criteria II result of the Taylor series expansion of $R(\tau)$ in the neighborhood of origin $(\tau \approx 0)$,

$$
R(\tau)=\left.\sum_{n=0}^{\infty} \frac{d^{n} R(\tau)}{d \tau^{n}}\right|_{\tau=0} \frac{\tau^{n}}{n !} .
$$

Due to the parity of $R(\tau)$ (stationary turbulence), the odd-order derivatives are null, in particular, $\left.\frac{d R(\tau)}{d \tau}\right|_{\tau=0}=0$. Therefore, from Eq. (20), it results as follows:

$$
R(\tau)=1+\left.\frac{1}{2} \frac{d^{2} R(\tau)}{d \tau^{2}}\right|_{\tau=0} \tau^{2}+O\left(\tau^{4}\right)
$$

and, consequently, $\left.\frac{1}{2} \frac{\mathrm{d}^{2} R(\tau)}{\mathrm{d} \tau^{2}}\right|_{\tau=0}<0$. The test of the second derivative in an interval centered at the origin $(\tau \approx 0)$ ensures a representation of $R(\tau)$ by quadratic polynomial and, at $\tau=0$, agrees with the first criteria.

Following [12], to introduce a time scale $\tau_{L}$, which contains temporal values close to the origin, the Taylor's autocorrelation function can be approximated by

$$
R(\tau) \approx 1-\frac{\tau^{2}}{\tau_{L}^{2}}
$$

meaning an osculating parabola $R(\tau)$ at origin. This expression defines the time scale $\tau_{L}$ by the relation

$$
\frac{1}{\tau_{L}^{2}}=-\left.\frac{1}{2} \frac{d^{2} R(\tau)}{d \tau^{2}}\right|_{\tau=0}
$$

(III) The Lagrangian integral time scale given by $T_{L}=\int_{0}^{\infty} R(\tau) d \tau$ is limited and well defined. It is a direct consequence of Criteria I.

(IV) From Wiener-Khintchin theorem [19], the $R(\tau)$ and $E(\omega)$ are expressed by a pair of Fourier cosine transform:

$$
R(\tau)=\frac{1}{\left\langle u^{2}\right\rangle} \int_{0}^{\infty} E(\omega) \cos (\omega \tau) d \omega
$$


and

$$
E(\omega)=\frac{2\left\langle u^{2}\right\rangle}{\pi} \int_{0}^{\infty} R(\tau) \cos (\omega \tau) d \tau
$$

where $\omega$ is the turbulent frequency.

According to the inertial subinterval theory (K41) [20], $E(\omega)$ can be expressed by $E(\omega)=\kappa \bar{\epsilon} \omega^{-2} \propto \omega^{-2}$, to $1 \ll \omega T_{L} \ll \frac{T_{L}}{\tau_{\eta}}$, where $\kappa$ is the dimensional universal constant, $\bar{\epsilon}$ is the average rate of turbulent energy dissipation, and $\tau_{\eta}$ is the Kolmogorov time scale.

\section{Author details}

Charles R.P. Szinvelski, Lidiane Buligon, Michel Baptistella Stefanello, Silvana Maldaner, Debora R. Roberti and Gervásio Annes Degrazia*

*Address all correspondence to: gervasiodegrazia@gmail.com

Universidade Federal de Santa Maria, Santa Maria, Brazil

\section{References}

[1] Mortarini L, Anfossi D. Proposal of an empirical velocity spectrum formula in low-wind speed conditions. Quarterly Journal of the Royal Meteorological Society. 2015;141:85-97

[2] Anfossi D, Alessandrini S, Trini Castelli S, Ferrero E, Oettl D, Degrazia G. Tracer dispersion simulation in low wind speed conditions with a new 2-D Langevin equation system. Atmospheric Environment. 2006;40:7234-7245

[3] Steeneveld GJ, Holtslag AAM. Meteorological aspects of air quality. In: Air Quality in the 21st Century. New York: Nova Science Publishers; 2009. pp. 67-114

[4] Mortarini L, Stefanello M, Degrazia G, Roberti D, Castelli ST, Anfossi D. Characterization of wind meandering in low-wind-speed conditions. Boundary-Layer Meteorology. 2016;161(1):165-182. DOI: 10.1007/s10546-016-0165-6

[5] Cava D, Mortarini L, Giostra U, Richiardone R, Anfossi D. A wavelet analysis of lowwind-speed submeso motions in a nocturnal boundary layer. Quarterly Journal of the Royal Meteorological Society. 2017;143:661-669. DOI: 10.1002/qj.2954

[6] Moor L, Degrazia G, Stefanello M, Mortarini L, Acevedo O, Maldaner S, Szinvelski C, Roberti D, Buligon L, Anfossi D. Proposal of a new autocorrelation function in low wind speed conditions. Physica A. 2015;438:286-292 
[7] Phillips P, Panofsky HA. A re-examination of lateral dispersion from continuous sources. Atmospheric Environment. 1982;16:1851-1860

[8] Mahrt L. Stably stratified atmospheric boundary layers. Annual Review of Fluid Mechanics. 2014;46:23-45

[9] Frenkiel FN. Turbulent diffusion: mean concentration distribution in a flow field of homogeneous turbulence. Advances in Applied Mechanics. 1953;3:61-107

[10] Manomaiphiboon K, Russell AG. Evaluation of some proposed forms of Lagrangian velocity correlation coefficient. International Journal of Heat and Fluid Flow. 2003;24 (5):709-712

[11] Tennekes H, Lumley JK. A First Course in Turbulence. Massachusetts: The MIT Press; 1972

[12] Hinze JO. Turbulence. New York: McGraw-Hill; 1975

[13] Tennekes $\mathrm{H}$. The exponential the Lagragian correlation function and turbulent diffusion in the inertial subrange. Atmospheric Environment. 1979;13(11):1565-1568

[14] McComb WD. The Physics of Fluid Turbulence. Oxford: Clarendon Press; 1990

[15] Kolmogorov AN. The local structure of turbulence in incompressible viscous fluid for very large Reynolds numbers. Doklady Akademii Nauk SSSR. 1941:299-303

[16] Tennekes H. Similarity relations, scaling laws and spectral dynamics. In: Atmospheric Turbulence and Air Pollution Modelling. Netherlands: Springer; 1984. pp. 37-68

[17] Degrazia G, Acevedo OC, Carvalho J, Goulart A, Moraes OLL, Campos Velho HF, Moreira DM. On the universality of the dissipation rate functional form and of the autocorrelation function exponential form. Atmospheric Environment. 2005;39(10):19171924

[18] Taylor GI. Diffusion by continuous movements. Proceedings of the London Mathematical Society. 1921;20:196-211

[19] Gardiner CW. Handbook of Stochastic Methods for Physics, Chemistry and the Natural Sciences. Berlin: Springer-Verlag; 1985. 442 p

[20] Kolmogorov AN. The local structure of turbulence in incompressible viscous fluid for large Reynolds number. Doklady Akademii Nauk SSSR; 1941;30(301):9-13 
